\title{
Wideband $4 \times 8$ Array Antennas with Aperture Coupled Patch Antenna Elements on LTCC
}

\author{
Dong Suk Jun ${ }^{1} \cdot$ Alexander Bondarik ${ }^{1} \cdot$ Hong-Yeol Lee ${ }^{1} \cdot$ Han-Cheol Ryu ${ }^{1} \cdot$

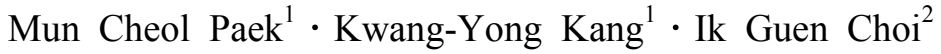

\begin{abstract}
We proposed a $4 \times 8$ array antenna with aperture-coupled patch antenna elements. The antenna was designed for 60 $\mathrm{GHz}$ operation and fabrication on the low-temperature cofired ceramic(LTCC) $\operatorname{substrate}\left(\varepsilon_{r}=5.8\right)$. The feedline with the stub was designed to enhance the radiating element bandwidth and the transition characteristics between the waveguide (WG) and microstrip line(MSL). Through the optimization of the antenna and feedline geometry, the antenna gain and the performance of the $10 \mathrm{~dB}$ bandwidth were $20.2 \mathrm{dBi}$ and $13 \%$ up, respectively. The measured results agreed with the simulated ones.
\end{abstract}

Key words : System on Package, $60 \mathrm{GHz}$ Array Antennas, Wideband Antenna.

\section{Introduction}

There is rapid growth demand for high-speed data transmission in wireless communications and for the miniaturization of components in high-frequency operation leads to facilitate the development of hardware and software for next-generation telecommunications. In particular, for unlicensed millimetre-wave bands such as $60 \mathrm{GHz}$ (all over the world), this growing demand has attracted the attention of both the research and industrial fields. The wide bandwidth allows the creation of communication and sensing systems that can easily support transmission rates above $1 \mathrm{Gbit} / \mathrm{s}^{[1],[2]}$.

The concept of system-on-package(SOP) has been developed to meet the requirements of system miniaturization. SOP implies a high integration of components, consisting of a system based on multilayer technology. The multilayer packaging technologies for SOP are necessary, and LTCC technology is used as the essential multilayer technology; it has become a popular technique for the production of highly integrated modules. These technologies are spot-lighted for their flexibility and diversity in realizing an arbitrary number of layers ${ }^{[3]}$.

Several types of the waveguide to microstrip-line transition have been reported. Array antennas, consisting of 16 parasitic microstrip antennas, have been discussed in ref. [4]. The antenna has characteristics of high gain and wide bandwidth. For the integration with other components of the system and for feeding of the antenna power, a rectangular type waveguide is used. The primary issue of this paper places the focus on the design of the feedline transition between waveguide(WG) and microstrip-line(MSL).

For the given conditions, the most suitable type of transition was waveguide to microstrip-line probe transition, where the micro-stripline extends into the waveguide through an aperture in the broad wall ${ }^{[5],[6]}$. In this solution, the critical view of transition is the aperture in the waveguide broad wall. Apertures should be made as small as possible to minimize the influence on the fields $(\mathrm{E}, \mathrm{H})$ that propagate inside the waveguide. On the other hand, the waveguide wall has a finite thickness and plays an essential role as the second ground plane for the inserted microstrip line. Hence, a stripline area with the length equal to the thickness of the waveguide was created. The effect was more significant at high frequencies where the wavelength of the signal became smaller. The reason for the effect may be explained as follows; in the wallaperture area of the small waveguide, there are actually two transitions: waveguide to stripline(SL) transition and stripline to microstrip-line(MSL) transition.

Transition by two transition media must provide impedance matching between the two transitions lines that maximizes the coupling instead of minimizing reflections. In addition to the impedance matching, a transition must provide an efficient field transition from one medium to another one by smooth and gradual change within the physical boundary conditions. To achieve a field and an impedance matching, step-by step transition or continuous taper transition is mainly used ${ }^{[7]}$.

Manuscript received April 14, 2010 ; revised August 24, 2010. (ID No. 20100414-11J)

${ }^{1}$ Electronics and Telecommunications Research Institute(ETRI), Daejeon, Korea.

${ }^{2}$ Department of Radio Engineering, Chungbuk National University, Chungju, Korea.

Corresponding Author : Dong Suk Jun (e-mail : dsjun@etri.re.kr) 
The main idea of this paper is to make the field transition more efficient for the waveguide to microstrip-line transition, by dividing the transition into three separated transitions, including waveguide to strip-line transition; strip-line to embedded microstrip-line transition, and embedded microstrip- line to microstrip-line transition.

Another complicated issue that was solved during the research that led to this paper was the packaging of all the transitions, and finally, integration with the microstrip antenna ${ }^{[8] \sim[10]}$. The authors of this paper designed and fabricated a $4 \times 8$ array antenna with aperture-coupled patch elements and waveguide to microstrip-line transition to obtain high gain and wide bandwidth.

\section{Waveguide to Microstrip Line Transition}

As shown in Fig. 1, rectangular waveguide(WG) to strip-line transition(SL) consisted of the WG being attached to the LTCC multilayer structure with SL. The LTCC structure under the WG was designed to realize effective energy transition. The number of layers is limited by the fabrication processes and should be appropriate to the number of layers for other nodes in the same system-ofpackage(SOP). For the described transition, the number of layers was seven (7) and the thickness of each layer was $0.1 \mathrm{~mm}$, with a relative permittivity of the LTCC layer $\varepsilon_{r}=5.9$, and tangent loss of the LTCC layer tan $\delta$ $=0.001$. In a simple way it can be thought that the SL has been inserted into the WG broad wall through the aperture. The vertical aperture size is equal to the SL thickness corresponding to impedance of $50 \Omega$. The WG part below SL forms the input section and the part under SL forms the short circuit section. The part of the SL inserted into the WG is the rectangular patch. The critical parameters for effective energy transition are patch dimensions and the size of the WG short circuit section.

The structure of the short circuit section was formed by adding three LTCC layers with metal covers on the

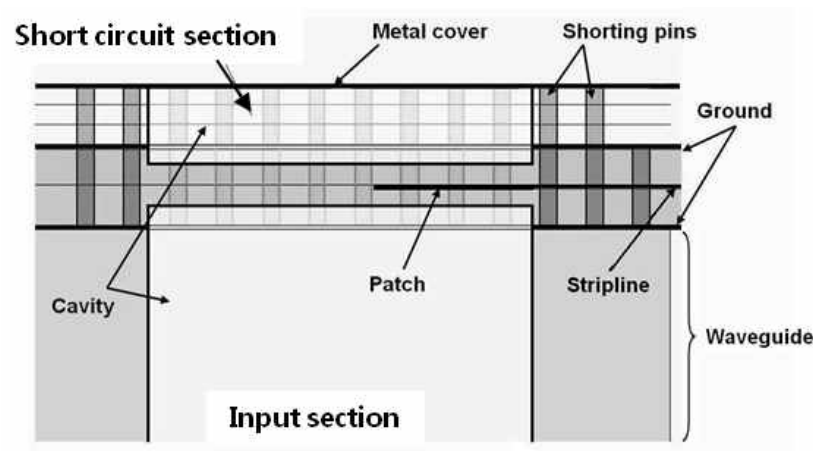

Fig. 1. Waveguide to strip-line transition side view.

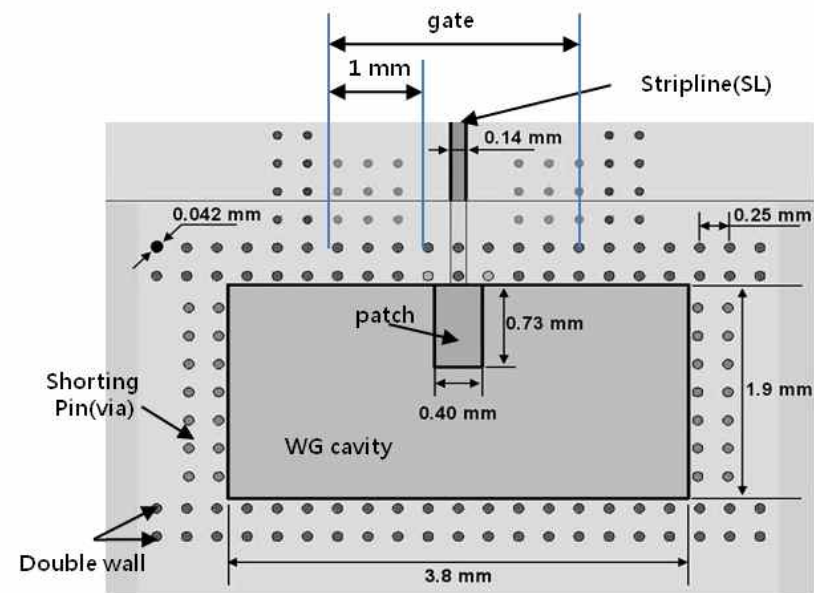

Fig. 2. Waveguide to strip-line transition top view.

tops(see Fig. 1). In all layers, the cavity of the WG length and width were made. Shorting pins were fabricated between adjacent metal layers through the LTCC layers. Metal layers and a double wall of pins simulated the metal walls. The arrangement of short circuit section pins is shown in Fig. 2.

Input and short circuit sections were in spaced alignment and the SL was in the gap between the two sections. The SL included ground planes on either side and apertures of the same size and shape as the WG cavity were cut into the ground planes. Between the input and short circuit sections, a double wall of pins simulated the continuation of the WG wallsGood simulation results have been achieved for the gate that has the four pin period size, which is $1 \mathrm{~mm}$. The shorting pins wall has been extended to prevent surface waves and has four periods size, in total, from the WG wall, which is $1 \mathrm{~mm}$ (see Fig. 2).

The dimensions of SL, patch, WG and shorting pins are shown in Fig. 2 also. Full-wave simulation scattering parameters related to designed transition are shown in Fig. 3.

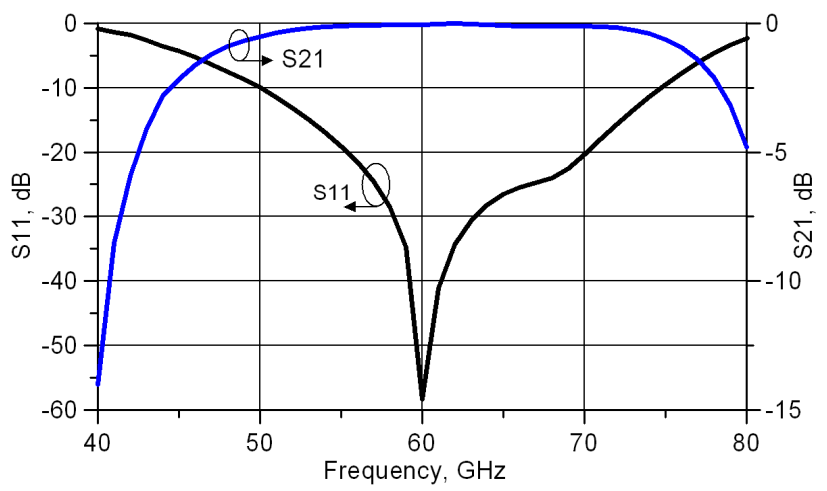

Fig. 3. Transition scattering parameters. 
The proposed SL to embedded microstrip line(EMSL) transition is shown in Fig. 4. The SL part and the EMSL part were positioned on the same level. EMSL ground, substrate and feedline were common for both types of feeding. 4 LTCC layers formed a substrate with the thickness of $0.4 \mathrm{~mm}$. The unique difference between two types of feedlines is that the SL has one more additional ground. The idea of the transition is to make a smooth shape cutting at the SL ground near the feedline in order to minimize return loss and to make energy transition effective. It is recognized also that along SL, the wall of shorting pins must play an important role to suppress the parallel plate modes. The most evident solution is to make round-shaped ground corners and to place shorting pins near the corners. Full-wave simulation, however, shows that this variant is not satisfactory.

The study described here proposed ellipse-shaped ground cutting and the view of this cutting type is shown in Fig. 4. The distance between the feedline and the shorting pins wall is fixed and represents the ellipse minor radius. The core variable to control for transition matching is the ellipse major radius.

EMSL impedance is approximately $60 \Omega$. Then, impedance mismatching can be compensated by the ellipseshaped cutting. Transition dimensions are shown in Fig. 4.

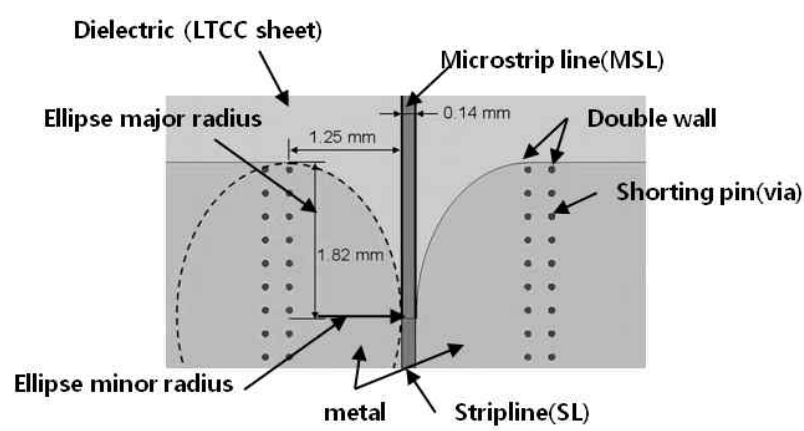

Fig. 4. Strip-line to embedded microstrip line transition(top view).

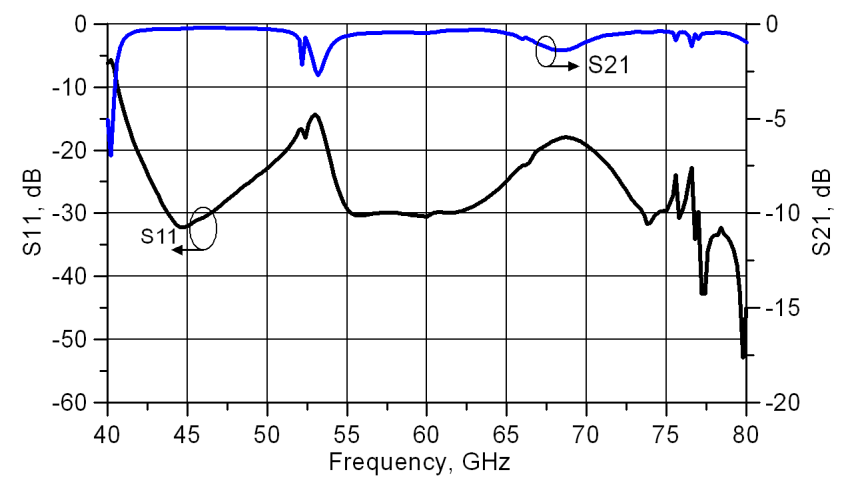

Fig. 5. Transition scattering parameters of EMSL.

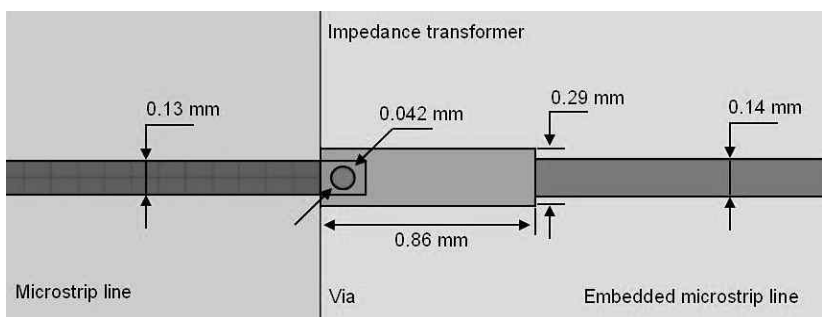

Fig. 6. Embedded microstrip line to microstrip line transition (top view).

Full-wave simulation scattering parameters for the designed transition are shown in Fig. 5.

The geometry of the proposed embedded microstrip line to microstrip line transition is shown in Fig. 6. MSL substrate thickness equals one LTCC layer with thickness of $0.1 \mathrm{~mm}$. Then, EMSL substrate thickness equals 4 LTCC layers with thickness of $0.4 \mathrm{~mm}$.

Two types of feedline have a common ground plane. MSL connects to the EMSL by means of via and via height is one LTCC layer. Impedance of MSL is about $60 \Omega$. The Quarter-wave impedance transformer has been used to compensate impedance difference between EMSL and MSL.

Dimensions of the EMSL to MSL transition are shown in Fig. 6 and full-wave simulation scattering parameters above transition are shown in Fig. 7.

After each separate transition was designed, all of them were assembled together and to form complex WG to EMSL transition. The top view of the complex transition is shown in Fig. 8. Full-wave simulation scattering parameters for the designed complex WG to EMSL transition are shown in Fig. 9. The designed complex transition shows wide bandwidth characteristics, about $38 \%$ greater, compared to the central frequency.

\section{The Aperture-Coupled Patch Antenna Structure}

We have designed the antenna with optimized band-

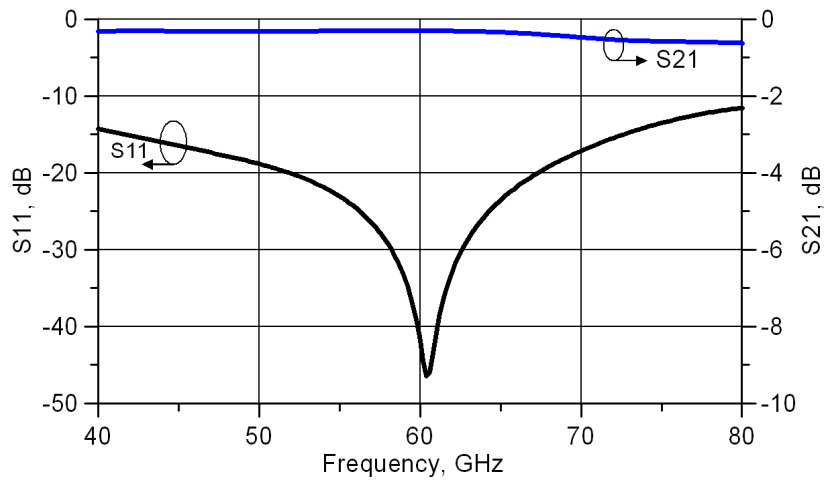

Fig. 7. Transition scattering parameters. 


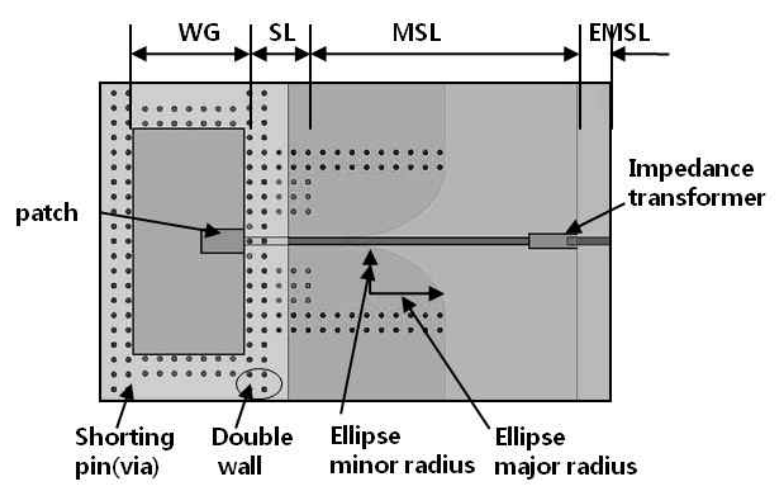

Fig. 8. Assembled transition(top view).

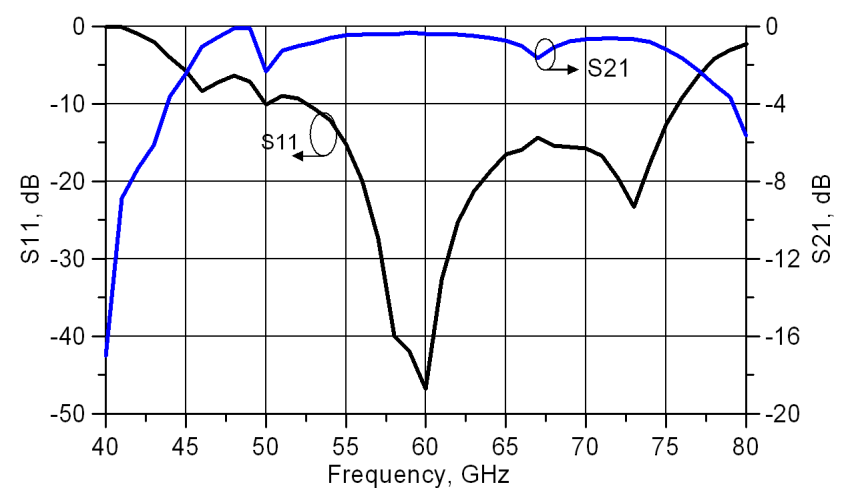

Fig. 9. Complex transition scattering parameters.

width, gain, side lobe level, cross polarization radiation, etc. The bandwidth is the key element to realize a highspeed data transmission and to implement a general-purpose antenna. A step-by-step optimization was carried out during the antenna design processes. Firstly, the aperture-coupled patch size and the distance between patch and feedline were optimized using the adjustment of the substrate thickness. As parameters have been changed within a broad range, the optimization to find the optimal parameters was performed using full-wave simulation software. The concept and core elements of the proposed aperture-coupled patch antenna are shown in Fig. 10.

The proposed antenna structure had three-layer LTCC substrates and the layer thicknesses were $h m, h f$, and $h p$ from bottom to top, respectively. A microstrip feedline arranged the patch antenna through an aperture slot in a ground.

The aperture-coupled patch antenna element had a rectangular structure. To control the feed-line impedance about $50 \Omega$, a set of parameters, which greatly influenced the antenna characteristics, such as substrate thickness, slot size, embedded microstrip stub length, and patch size, were optimized. In particular, to enhance the bandwidth of radiating elements, the feed-line with the stub was
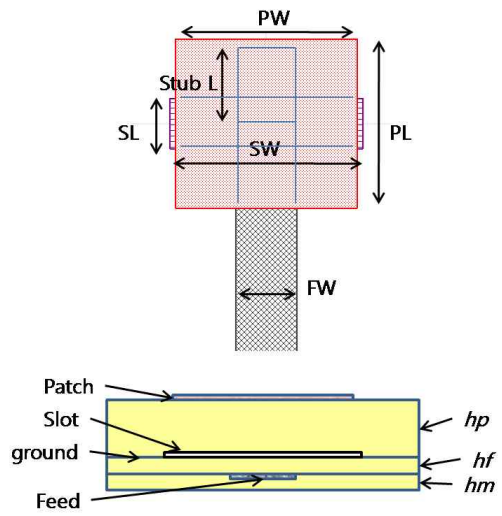

Fig. 10. The concept of the proposed aperture-coupled patch antenna elements.

designed and, in consideration of all of the antenna elements, array antennas with spacing, $d=2.3 \mathrm{~mm}=0.45 \lambda_{0}$ at $60.5 \mathrm{GHz}$ (where $\lambda_{0}$ is the wavelength in free space), were completed, as shown in Fig. 11. Table 1 shows the array antenna's physical dimensions, such as patch width (PW) of $720 \mu \mathrm{m}$ and length(PL) of $685 \mu \mathrm{m}$. The quarterwave transformer with length(tl1, tl3) and width (tw1, tw3) was used for impedance matching of antennas. The feed width(FW) and the height of each layer were 234 $\mu \mathrm{m}$ and $100 \mu \mathrm{m}$ respectively. On the basis of the single antenna structure, the array antennas were designed as shown in Fig. 11.

\section{Simulation and Measurement}

On the basis of the previous physical dimensions, return loss was simulated using Ansoft HFSS/designer. The design parameters were optimized to improve the characteristics of the transition $\left(S_{21}\right)$ and return loss $\left(S_{11}\right)$. The performance of the antennas was measured by an Anritsu 37169A vector network analyzer. Simulated and

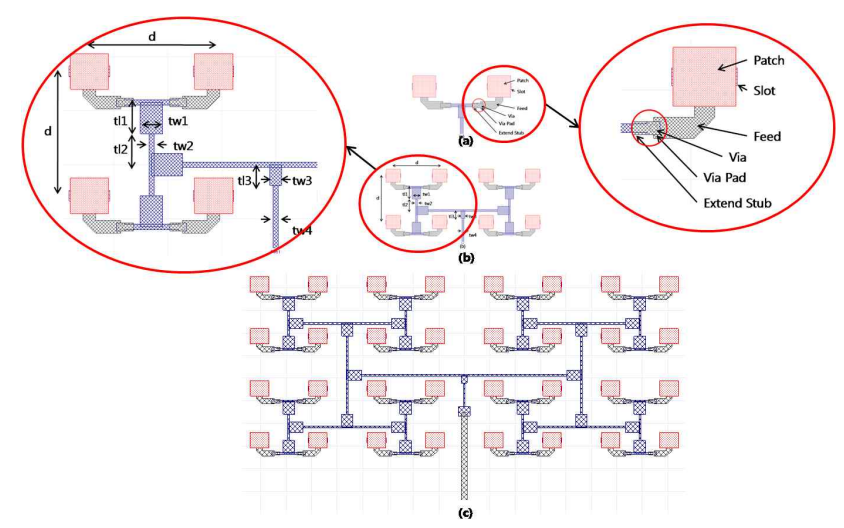

Fig. 11. The designed array antenna geometry including the feedline with stub. (a) $1 \times 2$ array antenna, (b) $2 \times 4$ array antenna, (c) $4 \times 8$ array antenna. 
Table 1. Array antennas physical dimension.

\begin{tabular}{|l|l|}
\hline \multicolumn{1}{|c|}{ Parameters } & Size(mm) \\
\hline Patch length(PL) & 0.685 \\
\hline Patch width(PW) & 0.72 \\
\hline Feed width(FW) & 0.234 \\
\hline Slot length(SL) & 0.231 \\
\hline Slot width(SW) & 0.75 \\
\hline Stub length(Stub L) & 0.32 \\
\hline Transformer width1(tw1) & 0.446 \\
\hline Transformer width2(tw2) & 0.214 \\
\hline Transformer width3(tw3) & 0.446 \\
\hline Transformer width4(tw4) & 0.214 \\
\hline Transformer length1(tl1) & 0.575 \\
\hline Transformer length2(t12) & 0.575 \\
\hline Transformer length3(tl3) & 0.575 \\
\hline Via diameter & 0.1 \\
\hline Via pad & 0.147 \\
\hline Extend stub & 0.224 \\
\hline Antenna element spacing(d) & 2.3 \\
\hline Height of each layer thickness & 0.100 \\
\hline
\end{tabular}

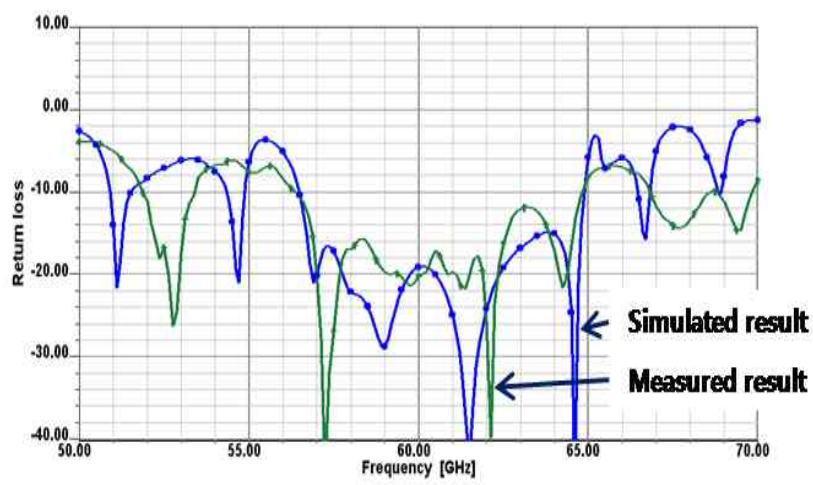

Fig. 12. The return loss for $4 \times 8$ the array antennas.

measured results are shown in Fig. 12. Especially, Fig. 13 and Fig. 14 represent the normalized radiation pattern of the $4 \times 8$ antenna array in E-plane and H-plane respectively. According to these results, it can be seen that the bandwidth of $10-\mathrm{dB}$ return loss is about $13 \%$ up for the central frequency range(from 57 to $65 \mathrm{GHz}$ ) and the gain is about $20.2 \mathrm{dBi}$. The side-lobe level was lower than $-15 \mathrm{~dB}$ in the H-plane and $-18 \mathrm{~dB}$ in the E-plane respectively. The half power beam width was about $8^{\circ}$ in the E-plane and $14.5^{\circ}$ in the H-plane, respectively. The gains of the antennas were about $20.2 \mathrm{dBi}$ in the frequency range(from 57 to $65 \mathrm{GHz}$ ).

The radiation pattern of H-plane has a small asymmetry because of the side lobes. These results could be sur-

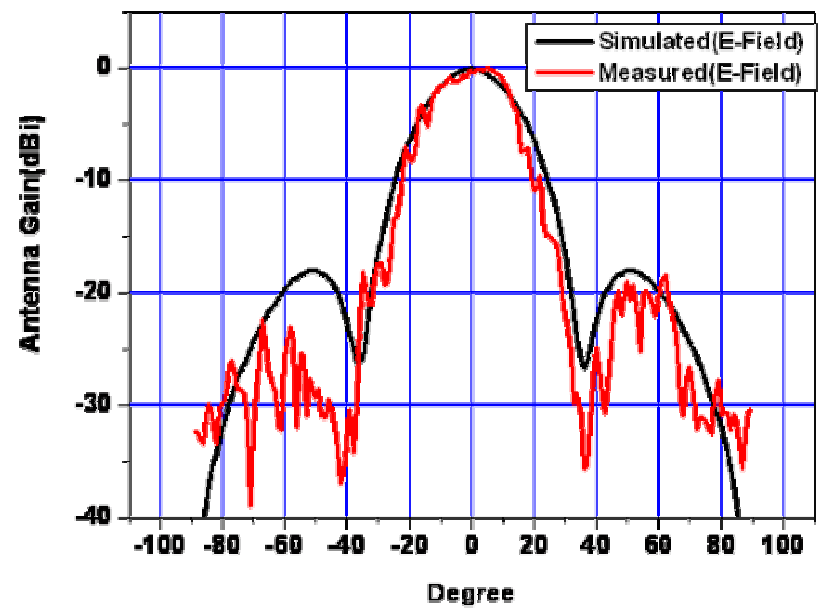

Fig. 13. The normalized radiation pattern(E-field) for the array antennas.

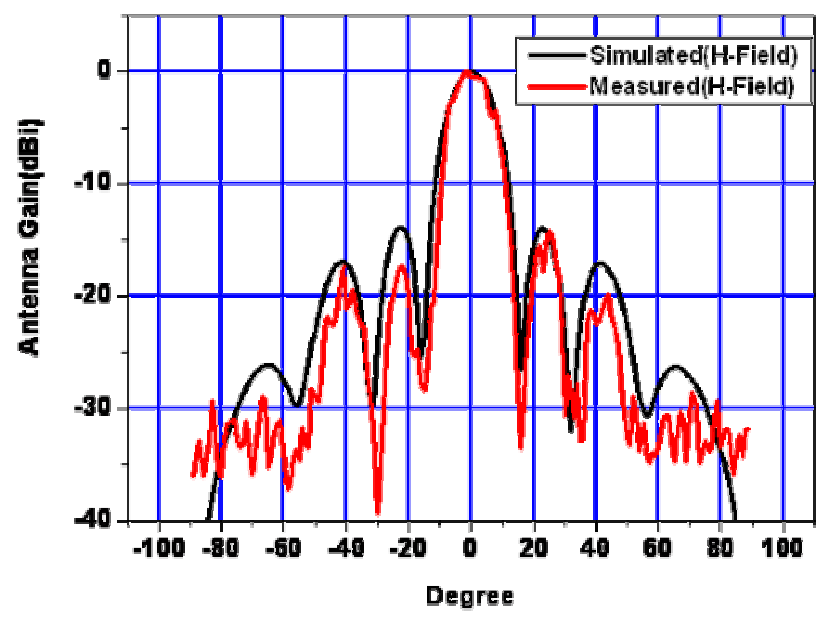

Fig. 14. The normalized radiation pattern(H-field) for the array antennas.

mised to be the result of the mutual coupling and asymmetry of the radiating patches connected with the fabricated feed network. The measured results are in a good agreement with simulated results. In the measured radiation pattern a noise was added because the measurement environment was not completely shielded. Fig. 15 shows the gain and cross polarization of the array antenna. The cross-polarization level was lower than $-22 \mathrm{dBi}$ for the broadside direction and the antenna gain was higher than $20.2 \mathrm{dBi}$.

Fig. 16 shows the front view and back view of the fabricated array antenna. The front view of the array antenna shows a simple design with the $4 \times 8$ array patch antenna. Several components such as the microstrip line (MSL) to embedded MSL(EMSL) transition, EMSL to strip-line(SL) transition and SL to waveguide(WG) transition are integrated on its back side. Specially, SL to 


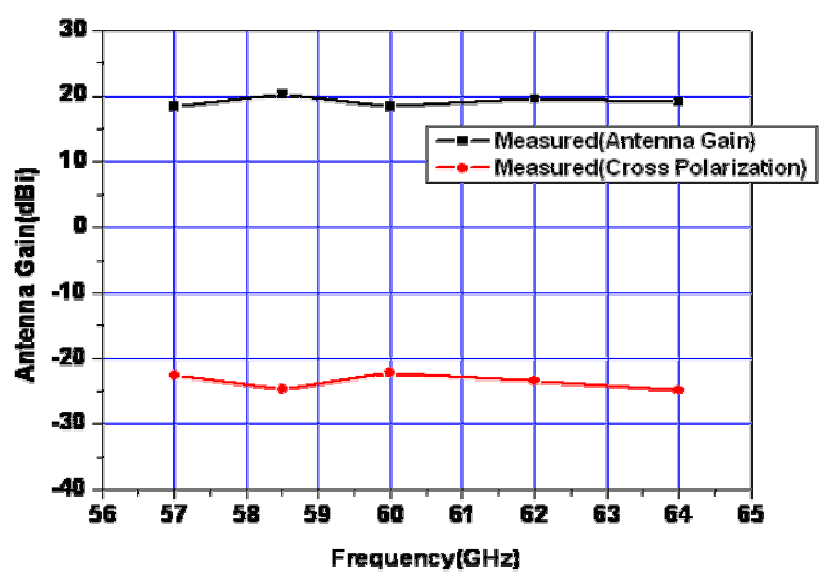

Fig. 15. The gain and cross polarization for the array antennas.

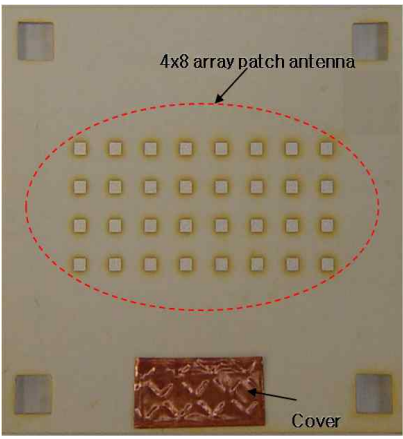

(a) Front view

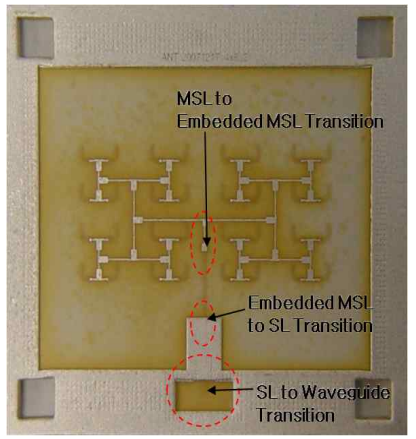

(b) Back view
Fig. 16. Fabricated array antennas.

WG transition connected with WR-15 is used to measure the radiation pattern and return loss.

\section{Conclusions}

The array antenna, based on LTCC substrate, operating at $60 \mathrm{GHz}$ was designed using full-wave simulation software. The wideband aperture-coupled patch antenna was proposed. The feed-line connected with the patch elements had a stub to broaden the bandwidth of the patch. After the optimization and the fabrication of array antennas with proposed antenna geometries, the measured results agreed well with the simulated ones. The fabricated array antennas on the LTCC substrate had a $20.2 \mathrm{dBi}$ gain the $13 \%$ up return loss bandwidth. The array antennas can be used in high speed wireless communications at the $60 \mathrm{GHz}$ band.

This work was supported by the IT R\&D program of MKE/KEIT [2006-S005-04, Development of THzwave oscillation/modulation/detection module and signal source technology].

\section{References}

[1] S. E. Gunnarsson, C. Kärnfelt, H. Zirath, R. Kozhuharov, D. Kuylenstierna, C. Fager, M. Ferndahl, B. Hansson, A. Alping, and P. Hallbjörner, "60 GHz single-chip front-end MMICs and systems for multi$\mathrm{Gb} / \mathrm{s}$ wireless communication", IEEE Journal of Solid State Circuits, vol. 42, pp. 1143-1157, May 2007.

[2] C. Daniels, R. W. Heath, "60 GHz wireless communications: Emerging requirements and design recommendations", IEEE Veh. Technol. Mag, pp. 41-50, Feb. 2007.

[3] K. Lim, S. Pinel, M. Davis, A. Sutono, C. -H. Lee, D. Heo, A. Obatoynbo, J. Laskar, E. M. Tantzeris, and R. Tummala, "RF-system-on-package(SOP) for wireless communications", IEEE Microwave Magazine, vol. 3, pp. 88-99, Mar. 2002.

[4] A. Bondarik, D. S. Jun, J. M. Kim, and J. H. Yun, "60 GHz system-on-package antenna array with parasitic microstrip antenna single element", in Proc. APMC 2008, Dec. 2008.

[5] Y. C. Shih, T. N. Ton, and L. Q. Bui, "Waveguideto-microstrip transitions for millimeter-wave applications", in IEEE MTT-S Int. Symp. Dig., vol. 1, pp. 473-475, 1988.

[6] K. Sakakibara, M. Hirono, N. Kikuma, and H. Hirayama, "Broadband and planar microstrip-to-waveguide transitions in millimeter-wave band", in Proc. ICMMT 2008, vol. 3, pp. 1278-1281, Apr. 2008.

[7] J. S. Izadian, S. M. Izadian, Microwave Transition Design, Artech House, 1988.

[8] Alexander Bondarik, Dong Suk Jun, Joung Myoun Kim, and Je Hoon Yun, "Investigation of microstrip antenna array stacked structure realized on LTCC for $60 \mathrm{GHz}$ band", Microwave and Optical Technology Letters, vol. 52, no. 2, Feb. 2010.

[9] Dong Suk Jun, Alexander Bondarik, Hong Yeol Lee, IK Guen Choi, Mun Cheol Paek, and Kwang Yong Kang, "Design of an array antenna with aperture coupled patch elements on LTCC", Global Symposium on Millimeter Waves 2010, pp. 177-180, Apr. 2010.

[10] Hong Yeol Lee, Young Goo Kim, Dong Suk Jun, Ik Guen Choi, Kang Ho Park, and Jong dae Kim, "Broadband transition between microstrip line and WR15 rectangular waveguide using low temperature cofired ceramic process", Microwave and $O p$ tical Technology Letters, vol. 52, no. 3, Mar. 2010. 


\section{Dong Suk Jun}

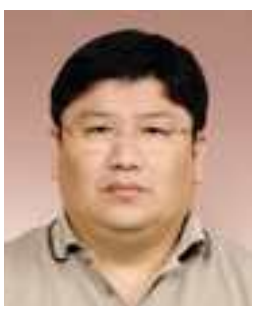

is a principal member of the engineering staff and a team head of the Tera-electronics Device Team of ETRI. He received a BS degree in electronics engineering from Hanbat National University, Daejeon, Korea, and a M.S. degree in electrical engineering from Chungbuk National University, Chungju, Korea, in 1996. Since 1984, he has been working with ETRI. His research interests include the microwave/millimeter/terahertz-wave passive component design, antenna design, beamforming system and radiometer $\mathrm{He}$ is currently working on the terahertz materials, devices/components, and systems.

\section{Alexander Bondarik}

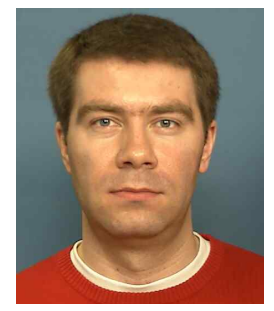

received M.Sc. degree in applied mathematics and physics from the Moscow Institute of Physics and Technology(MIPT) in 2003. In 2000, as research fellow, he joined an antenna team in JSC "Radiophyzika" in Moscow. From May 2006 to April 2010 he worked for Electronics and Telecommunications Research Institute (ETRI) in South Korea, where he developed antenna array for $60 \mathrm{GHz}$ and antenna measurements techniques. He is currently working toward a Ph.D. degree at Lund University, Sweden, at the Electrical and Information Technology Department. He is involved in a $60 \mathrm{GHz}$ high speed wireless communication project.

\section{Hong-Yeol Lee}

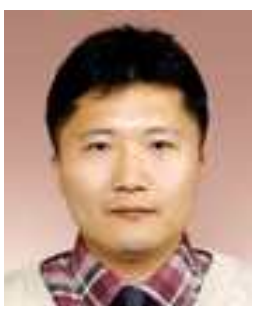

received B.S., M.S., and Ph.D. degrees in radio engineering from Chungbuk National University, Chungju, Korea, in 1999, 2001, and 2005, respectively. Since 2000, he has been working with ETRI, Daejeon, Korea, where he currently works on a RF satellite payload research team as a senior member of the engineering staff. His current research areas include $\mathrm{RF} /$ millimeter-wave passive component design, antenna design and satellite system engineering.

\section{Han-Cheol Ryu}

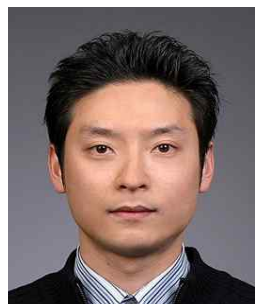

received a B.S. degree in electronic engineering from Hanyang University, Seoul, Korea, in 2000, and a M.S. degree from Pohang University of Science and Technology, Pohang, Korea, in 2002. Since 2002, he has been working with ETRI on the development of microwave tunable devices based on ferroelectric thin films and the THz-TDS system. His current research activities are in the development of a continuous terahertz photomixing system based on two DFB-LDs.

\section{Mun Cheol Paek}

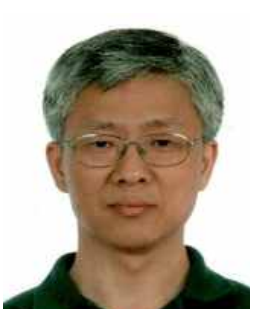

is a principal member of the engineering staff with the Tera-electronics Device Team of ETRI. He received a B.S. degree from Seoul National University, Seoul, in 1979, and M.S. and Ph.D. degrees in materials science from Korea Advanced Institute of Science and Technology(KAIST), Seoul, in 1982 and 1990, respectively. Since 1982, he has been a member of the engineering staff with ETRI. From 1996 to 1997, he was associated with the Lucent Technologies Bell-Labs, Murray Hill, where he was a visiting scientist in the physical science research group. His research interests include electronic materials and characterization technologies, optical data storage systems, and terahertz technologies. He is currently working on terahertz materials, components, and systems.

\section{Kwang-Yong Kang}

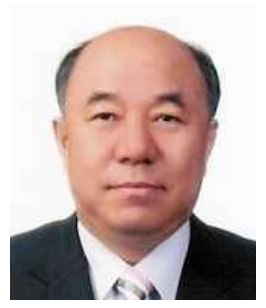

is a principal member of the engineering staff and a team head of the Teraelectronics Device Team of ETRI. He received a B.S. degree from Seoul National University, Seoul, Korea, in 1975, and his Ph.D. degree in solid-state physics from Pusan National University, Busan, Korea, in 1988. Since 1989, he has been a member of the engineering staff of the ETRI, Daejeon, Korea. He is a member of the IEEE MTT and OSA of USA, JJAP of Japan, and KEES/KPS of Korea. His research interests include the HTS thin films and microwave/millimeter-wave device, metal-insulator transition(MIT) devices, and terahertz technologies. He is currently working on the terahertz materials, devices/components, and systems. 


\section{Ik-Guen Choi}

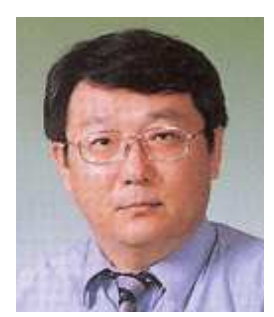

joined the E.E. faculty in Chungbuk National University in 1994. Before joining this university, he was a graduate research associate at the Electro-Science Laboratory of The Ohio State University, Columbus, Ohio from December 1982 to August 1986 and researched on the underground radar development project and the microstrip antenna analysis project. From September 1986 to August 1987, he was a researcher at the antenna laboratory of the University of Massachusetts, Amherst, M.A., where he researched in the development of microstrip array antenna design tools. From September 1987 to August 1993, he was a senior researcher at the Electronics and Telecommunication Research Institute, Korea, where he was involved, as head of the radio engineering section, in the development of mobile communication systems and in the development of the measurement methodology of electromagnetic compatibilities. He had developed the vehicle-mounted Satellite TV broadcasting receiving antenna systems in 1996. He is currently working on the $900 \mathrm{MHz}$ RFID reader development and small tag antenna design at 13 $\mathrm{MHz}$ and $900 \mathrm{MHz}$. RFID reader development research includes developments of the diversity antennas, RF module and digital hardware module with relevant DSP/CPU software. 